cases were reported as indefinite for dysplasia, 32/620 were low grade dysplasia and 10/620 cases were classified as high grade dysplasia. $233 / 620$ (37.6\%) patients had on average one follow-up and 100/620 (16.1\%) had two or more follow ups during the study period.

Conclusion Compliance with BSG follow-up recommendations and other practise parameters is poor. We recommend a formal surveillance programme with dedicated endoscopy lists to improve compliance and permit a meaningful assessment of the clinical and cost effectiveness of such strategy.

Disclosure of Interest None Declared

\section{PTU-154 SIRT2 MODULATES THE INFLAMMATORY RESPONSE IN OESOPHAGEAL ADENOCARCINOMA}

doi:10.1136/gutjnl-2013-304907.244

1."L K E Schulz, 'P Lao-Sirieix, ${ }^{2} \mathrm{M}$ 0' Donovan, 'R C Fitzgerald. 'MRC Cancer Cell Unit ${ }^{2}$ Department of Histopathology and Cytology, Addenbrooke's Hospital, University of Cambridge, Cambridge, UK

Introduction Gastro-oesophageal reflux disease is the main risk factor for Barrett's oesophagus (BE), the precursor lesion to oesophageal adenocarcinoma. In BE, GORD leads to chronic inflammation and to NF- $\kappa \mathrm{B}$ pathway activation.

SIRT2 is a histone deacetylase involved in deacetylation of key players in the cell, including p65, one subunit of the NF- $\kappa B$ transcription complex. SIRT2 is part of our previously published gene signatures in which loss of SIRT2 confers a poor prognosis and overexpression a good prognosis in keeping with its known role as a tumour suppressor.

We hypothesised that exerts its protective effect through recruitment of inflammatory cells to the tumour site via the NF- $\mathrm{KB}$ pathway The aim of this study was to assess the inflammatory infiltrate in positive tumoursto assess the relationship betweenthe NF- $\mathrm{BB}$ pathway.

Methods 76 surgical resection specimen of oesophageal adenocarcinoma were immunostained for SIRT2. An in-depth analysis of the nature of inflammatory cells localised to high SIRT2 areas was done in 5 cases using immune cell markers (CD3, CD4, CD8, CD20, CD56 and CD68). NF- $\kappa B$ and SIRT2 luciferase reporter assays were used with SIRT2 overexpression and TNF $\alpha$ stimulation to study the interplay between the NF- $\kappa B$ pathway and SIRT2. A panel of SIRT2 promoter mutants with mutations of one or two or both $\mathrm{NF}-\mathrm{KB}$ putative binding sites, identified through an in silico analysis, were also used.

Results $32 \%$ of the cases were strongly positive for SIRT $2(+3$ and +2 on a scale from 0 to +3 where 0 is negative). A higher number of inflammatory cells were identified in SIRT2-positive cases compared to SIRT2 negative cases. In particular, SIRT2 positive cases showed strong staining for CD68 indicating an enrichment in the number of macrophages. SIRT2 overexpression significantly downregulated NF- $\kappa B$ activity $(p=0.0011)$. Immunoblotting suggests that this downregulation is probably conferred by the deacetylation of Lysine 310 at the p65 subunit of NF- $\kappa B$. Luciferase assays with the full-length SIRT2-promoter reporter revealed that the SIRT2 promoter was induced by TNF $\alpha$ stimulation (activates NF- $\kappa B$ pathway). This stimulation resulted in decreased luciferase activity when the NF- $\kappa B$ binding sites mutants were used, suggesting a direct action of NF- $\kappa B$ on SIRT2.

Conclusion In oesophageal adenocarcinoma, SIRT2 expression is linked with an increased inflammatory infiltrate, especially macrophages. Luciferase reporter assays suggest that SIRT2 and NF- $\mathrm{KB}$ regulate each other. Taken together, downregulation of NF- $\kappa B$ by SIRT2 could be an explanation for the protective effect of SIRT2 overexpression in oesophageal adenocarcinoma. Further work is required to confirm these findings.

Disclosure of Interest None Declared

\section{PTU-155 LESSONS LEARNT FROM THE FIRST 50 CONSECUTIVE PRIMARY LAPAROSCOPIC NISSEN FUNDOPLICATIONS IN A SINGLE SURGEON'S PRACTICE}

doi:10.1136/gutjnl-2013-304907.245

1."L Sanyaolu, 'L Cleveland, 'A Rasheed. 'Gwent Institute for Minimal Access Surgery, Newport, UK

Introduction Laparoscopic Nissen fundoplication (LNF) is widely used in the surgical management of gastroesophageal reflux disease (GORD). However, it is a complex operation that requires advanced laparoscopic skills. Very few studies report objective testing postoperatively; those that do show high rates of failure within the first 1-3 years following surgery. Complications and failures of LNF are directly related to surgeon's experience and the learning curve has been identified as a confounding factor.

The Aim of this is analyse a single surgeon's first 50 consecutive primary LNFs experience. The data will be used to attempt to define the learning curve (LC) for LNF using success as surrogate marker of competency, and of how this may influence future training.

Methods All the patients who underwent antireflux surgery were entered into a prospectively maintained database. The procedures were performed using a five-trocar technique and with $10-/ 5-\mathrm{mm}$ ports and instruments. Surgical outcome was recorded using the Viscik symptom evaluation tool and complications graded according to the Dindo-Clavien classification. Captured parameters included patients' demographics, BMI, ASA grade, pre-operative investigations, operating time, indications for surgery, laparoscopic to open conversion rates, re-operation rates, morbidity, and mortality, follow up, and further investigations and interventions. Systematic case per case retrospective note analysis was performed.

Results The first fifty consecutive cases underwent primary sutured cruroplasty and Nissen's fundoplication by or under the direct supervision of the operating surgeon. One patient was abandoned due to inability to access the hiatus and one converted to open for bleeding from the omentum upon insertion of the primary port (both were during the first 25 cases). Three patients suffered with complete post-operative dysphagia, 2 resolved during the first 48 hours and one was converted to Toupet's (they were all during the first 25 cases). On follow up, one patient was re-operated and undone two years following the procedure for continuous epigastric pain with good outcome and one who had belching as a predominant symptom did not derive any symptomatic benefit from the procedure.

Conclusion Laparoscopic antireflux surgery "a reparative procedure" is not a natural extension of laparoscopic cholecystectomy "an extirpative procedure". Different dissecting skills and mastery of intracorporeal suturing and knot tying are necessary for laparoscopic antireflux surgery. The long and steep learning curve can be modified but not eliminated by systematic training and direct supervision during the first 25 cases. Occasional surgical treatment of GORD must be discouraged in order to achieve best possible surgical outcomes.

Disclosure of Interest None Declared

\section{PTU-156 THE COMBINATION OF AUTOFLUORESCENCE IMAGING AND PROBE-BASED CONFOCAL LASER ENDOMICROSCOPY HAS AN EXCELLENT DIAGNOSTIC ACCURACY FOR DYSPLASIA IN BARRETT'S ESOPHAGUS}

doi:10.1136/gutjnl-2013-304907.246

1." $\mathrm{M}$ Di Pietro, 'E Bird-Lieberman, 'X Liu, ${ }^{2} \mathrm{M}$ O'Donovan, 'R C Fitzgerald. 'Hutchison MRC Research Centre; 2Pathology, Cambridge University Hospitals, Cambridge, UK

Introduction Endoscopic surveillance in Barrett's oesophagus (BO) has major limitations including sampling error due to inconspicuous dysplasia, need for multiple biopsies and subjectivity of pathological assessment of dysplasia. Probe-based confocal laser 